\title{
THE CHEMICAL INHOMOGENEITY OF FAINT M13 STARS: CARBON AND NITROGEN ABUNDANCES ${ }^{1}$
}

\author{
Michael M. Briley, ${ }^{2}$ Judith G. Cohen, ${ }^{3}$ And Peter B. Stetson ${ }^{4}$ \\ Received 2003 October 14; accepted 2003 December 11
}

\begin{abstract}
Building upon earlier observations that demonstrate substantial star-to-star differences in the carbon abundances of M13 subgiants, we present new Keck LRIS spectra reaching more that 1.5 mag below the M13 mainsequence turnoff (to $V \approx 20$ ). Our analysis reveals a distribution of $\mathrm{C}$ abundances similar to that found among the subgiants, implying little change in the compositions of the M13 stars at least through the main-sequence turnoff. We presume these differences to be the result of some process operating early in the cluster history. Additional spectra of previously studied bright M13 giants have been obtained with the $5 \mathrm{~m}$ Hale Telescope. A comparison of $\mathrm{C}$ abundances derived using the present methods and those from the literature yield a mean difference of $0.03 \pm 0.14$ dex for four stars in common with the 1996 study by Smith et al. and $0.14 \pm 0.07$ dex for stars also observed in Suntzeff's 1981 survey (if one extreme case is removed). We conclude that the lower surface C abundances of these luminous giants as compared with the subgiants and main-sequence stars are likely the result of mixing rather than a difference in our abundance scales. NH band strengths have also been measured for a handful of the most luminous M13 turnoff stars. While molecular band formation in such stars is weak, significant star-to-star NH band strength differences are present. Moreover, for the stars with both $\mathrm{C}$ and $\mathrm{N}$ measurements, differences between stars in these two elements appear to be anticorrelated. Finally, the most recent $\mathrm{C}$ and $\mathrm{N}$ abundances for main-sequence, main-sequence turnoff, and subgiant stars in 47 Tuc, M71, M5, and the present M13 data are compared.
\end{abstract}

Key words: globular clusters: general — globular clusters: individual (M13) — stars: abundances — stars: evolution

On-line material: machine-readable tables

\section{INTRODUCTION}

It has been known since the early 1970s that the otherwise indistinguishable members of any given Galactic globular cluster exhibit significant star-to-star variations in surface abundances of certain light elements (most notably $\mathrm{C}$ and $\mathrm{N}$, as well as $\mathrm{O}$, and often $\mathrm{Na}, \mathrm{Al}$, and $\mathrm{Mg}$ ). ${ }^{5}$ However, while the abundance patterns commonly observed point to an origin in proton-capture nucleosynthesis (Denissenkov \& Denissenkova 1990; Langer \& Hoffman 1995; Cavallo, Sweigart, \& Bell 1996), identification of the specific reaction site or sites and a full theoretical description of the abundance-modifying processes remain uncertain. As has been pointed out in numerous reviews (see Smith 1987; Kraft 1994; Da Costa 1998), two possibilities exist.

First, the present-day cluster stars may have modified their own surface compositions through some mixing process not included in standard models (i.e., an "in situ" scenario). By

\footnotetext{
${ }^{1}$ Based in large part on observations obtained at the W. M. Keck Observatory, which is operated as a scientific partnership among the California Institute of Technology, the University of California, and the National Aeronatics and Space Administration. The Observatory was made possible by the generous financial support of the W. M. Keck Foundation.

2 Department of Physics and Astronomy, University of Wisconsin Oshkosh, 800 Algoma Boulevard, Oshkosh, WI 54901; mike@maxwell.phys.uwosh.edu.

3 Palomar Observatory, MS 105-24, California Institute of Technology, Pasadena, CA 91125.

${ }^{4}$ Dominion Astrophysical Observatory, Herzberg Institute of Astrophysics, National Research Council of Canada, 5071 West Saanich Road, Victoria, BC V9E 2E7, Canada.

5 Note that we are excluding $\omega$ Cen and M22, both of which appear to have experienced some degree of self-enrichment.
}

far the most promising candidate site in this regard is the region above the H-burning shell after first dredge-up in evolving cluster giants, where conditions for partial $\mathrm{CN}$ - and possibly ON-cycle reactions exist (see Sweigart \& Mengel 1979 for one of the earliest treatments). Subsequent circulation of this material into the stellar envelope via meridional currents or turbulent diffusion (see, e.g., Denissenkov \& VandenBerg 2003) will result in decreasing $C$ abundances and increasing $\mathrm{N}$ with evolutionary state, as has been observed along the red giant branches (RGBs) of several metal-poor clusters (for classic examples, see Carbon et al. 1982; Trefzger et al. 1983; Briley et al. 1990). Moreover, the operation of such a mechanism can at least qualitatively explain the $\mathrm{O}$ and $\mathrm{Mg}$ versus $\mathrm{Na}$ and $\mathrm{Al}$ anticorrelations found among the most luminous red giants in several clusters (e.g., Kraft et al. 1998 and references therein).

Common to all models of this process is the prohibition of "extra mixing" by the molecular-weight gradient left behind by the inward excursion of the convective envelope during first dredge-up. Only after the molecular-weight discontinuity has been destroyed by the outward-moving H-burning shell, an event marked by the RGB luminosity function (LF) bump, is mixing expected to take place (see, e.g., Sweigart \& Mengel 1979; Charbonnel, Brown, \& Wallerstein 1998). This theoretical prediction appears to be borne out by observations of decreased $\mathrm{Li}$ abundances following the LF bump in NGC 6752 (Grundahl et al. 2002) and similar drops in ${ }^{12} \mathrm{C} /{ }^{13} \mathrm{C}$ seen by Shetrone (2003) in NGC 6528 and M4.

However, this cannot be the entire picture. As early as 1978, it was noted by Hesser (1978) that the subgiant branch (SGB) and likely the main-sequence (MS) stars of 47 Tuc also possessed star-to-star differences in $\mathrm{CH}$ and $\mathrm{CN}$ band strengths. 
This has been most recently followed in 47 Tuc to $\approx 2.5$ mag below the MS turnoff (MSTO) by Harbeck, Smith, \& Grebel (2003). An analysis of their observed $\mathrm{CN}$ and $\mathrm{CH}$ band strengths yields factor of 10 variations in $\mathrm{N}$ anticorrelated with factor of 3 differences in C (Briley et al. 2004), matching those found among the more evolved members. Such CN and $\mathrm{CH}$ ( $\mathrm{N}$ and $\mathrm{C}$ ) variations have also been shown to exist among the MS, MSTO, and/or SGB stars of NGC 6752, M71, and M5 (Suntzeff \& Smith 1991; Cohen 1999b; Cohen, Briley \& Stetson 2002). Moreover, star-to-star variations in $\mathrm{Na}, \mathrm{Al}, \mathrm{O}$, and $\mathrm{Mg}$, similar to those found among the luminous cluster stars, have been identified among the SGB and MSTO stars of 47 Tuc (Briley et al. 1996), NGC 6752 (Gratton et al. 2001), M71 (Ramírez \& Cohen 2002), and M5 (Ramírez \& Cohen 2003). Although the various correlations and anticorrelations among these elements suggest the presence of material exposed to proton-capture reactions, such stars lie well below the LF bump, and particularly in the case of the MS stars, no mechanism is known for circulating significant quantities of $\mathrm{CN}(\mathrm{O})$-nucleosynthesized material to their surfaces.

Thus there is the second possible origin of the GC abundance variations - they have been set in place before RGB ascent and are due to the operation of some mechanism early in the cluster history (sometimes referred to as a "primordial" scenario). A number of possibilities exist, as are discussed extensively by Cannon et al. (1998), including that the protocluster gas was inhomogeneous in these elements (a true primordial origin), that there was an extended period of star formation of sufficient duration to allow some low-mass stars to form with material ejected from more massive, alreadyevolved cluster asymptotic giant branch (AGB) stars, and that the present-day cluster stars have accreted AGB ejecta onto their surfaces after their formation. The appeal of AGB stars as sites of the proton-capture nucelosynthesis lies in their ability to modify the cluster gas in light elements (including $\mathrm{C}, \mathrm{N}, \mathrm{O}$, $\mathrm{Al}, \mathrm{Na}$, and $\mathrm{Mg}$; see Ventura et al. 2001) while not altering the abundances of heavy elements.

As the reader has likely noted, observational evidence exists for both mixing and early contamination scenarios, which has led many investigators to conclude that the compositions of the cluster stars we observe today are not the result of one or the other scenario exclusively, but rather both. Unfortunately, this leads to difficulties in disentangling the contributions of each process among the more luminous cluster stars-a problem that can only be reconciled by exploring the compositions of a cluster's stars to the MSTO and below. Clearly, abundance trends found among a cluster's MS stars reflect the original makeup of the bright giants, while deviations from this "baseline" composition are likely the result of mixing. This was recently demonstrated in the case of M13 by Briley, Cohen, \& Stetson (2002, hereafter BCS02) - that a large spread in $\mathrm{C}$ abundances exists among the SGB stars of M13, which presumably reflects star-to-star variations in $\mathrm{C}$ abundances set early in the cluster history. However, the SGB carbon abundances also appear larger than those found by other investigators among the more luminous M13 stars, implying the operation of a mixing mechanism on the RGB that has reduced surface $\mathrm{C}$ abundances.

In the present paper, we return to M13 and extend our sample more than 2 mag fainter to include MS stars. In addition, we have also obtained spectra of M13 bright giants observed in earlier studies to verify our abundance scale. Our results confirm those of $\mathrm{BCS} 02$ - that a primordial spread in the distribution of light elements exists in M13 that has further been modified during RGB ascent. Measurements of the $3360 \AA \mathrm{NH}$ bands also were obtained for a handful of the more luminous stars in our sample. Nitrogen abundances calculated from these bands suggest a $\mathrm{C}$ versus $\mathrm{N}$ anticorrelation at the level of the MSTO.

\section{OBSERVATIONS}

\subsection{The Faint Star Sample}

The initial sample of stars in M13 was aimed to produce subgiants at the base of the RGB. It consisted of those stars from the photometric database (described by Stetson, Hesser, \& Smecker-Hane 1998; Stetson 2000) located more than $150^{\prime \prime}$ from the center of M13 (to avoid crowding) with $16.9<V<17.35$ and with $0.86<V-I<0.96$. A slit mask with 0.7 wide slitlets, narrower than normal to enhance the spectral resolution and minimize contributions from adjacent stars in these crowded fields, was designed using J. G. C.'s software from this sample and used in 2001 May with the Low Resolution Imaging Spectrometer (LRIS; Oke et al. 1995) at Keck. For this slit mask, as for all those used for the M13 stars, the red side of LRIS was set to include the $\mathrm{Na} D$ lines and $\mathrm{H} \alpha$. We used the highest possible dispersion, $0.64 \AA$ $\operatorname{pixel}^{-1}\left(29 \mathrm{~km} \mathrm{~s}^{-1}\right.$ pixel $\left.^{-1}\right)$, or $1.9 \AA$ per spectral resolution element there, to facilitate radial velocity confirmation of cluster membership. Given that the radial velocity of M13 is $-246 \mathrm{~km} \mathrm{~s}^{-1}$, distinguishing field stars from cluster members is then straightforward.

The blue side of LRIS (McCarthy et al. 1998) was used with the 600 line $\mathrm{mm}^{-1}$ grism blazed at $5000 \AA$. The detector for LRIS-B at that time was a $2048 \times 2048$ CCD not optimized for UV response. The spectra covered the range from $\sim 3400$ to $5000 \AA$, thus including the strongest $\mathrm{CN}$ band at $3885 \AA$ and the $\mathrm{G}$ band of $\mathrm{CH}$ at $4300 \AA$, with a resolution of $\sim 4 \AA\left(1.0 \AA\right.$ pixel $\left.^{-1}\right)$. Two additional slit masks were defined from this sample and used in 2002 May during less than ideal weather conditions for six exposures of $4800 \mathrm{~s}$ each. The spectra were dithered by moving the stars along the length of the slitlets by $2^{\prime \prime}$ between exposures. These spectra are part of those presented in BCS02.

Because of the crowded fields, in addition to the intended stars some slitlets contained additional stars bright enough to provide suitable spectra, and these were utilized as well. As might be expected from the LF, most of the secondary sample consists of stars at or just below the MSTO. Hence, subtraction of sequential exposures was not possible; they were reduced individually using Figaro (Shortridge 1988) scripts, and then the one-dimensional spectra for each object were summed.

Based on the serendipitous main-sequence stars found in the 2002 observations (see the plots in BCS02), we decided to try to reach main-sequence stars well below the turnoff in M13, sufficiently faint to be cool enough to have detectable $\mathrm{CH}$ bands. The criteria used to define the sample from the photometric database were $19.3<I<19.7, V-I$ within $0.06 \mathrm{mag}$ of the MS of M13, taken as $1.26+0.28(I-19.4)$, and located more than $200^{\prime \prime}$ from the center of M13. A single slit mask with 0.8 wide slitlets was designed and used at Keck with LRIS on 2003 June 26 . The blue spectra cover the full range from the atmospheric cutoff to $5000 \AA$, with $1.0 \AA$ pixel $^{-1}$ and a spectral resolution of $\sim 4 \AA$. Four exposures totaling $4200 \mathrm{~s}$ were obtained. The new very high quantum efficiency detector for LRIS-B, consisting of two $2 \mathrm{~K} \times 4 \mathrm{~K}$ Marconi CCDs, with $15 \mu \mathrm{m}$ pixels and a readout noise of $4.0 e^{-}$, was completed and installed into LRIS in 2002 June 


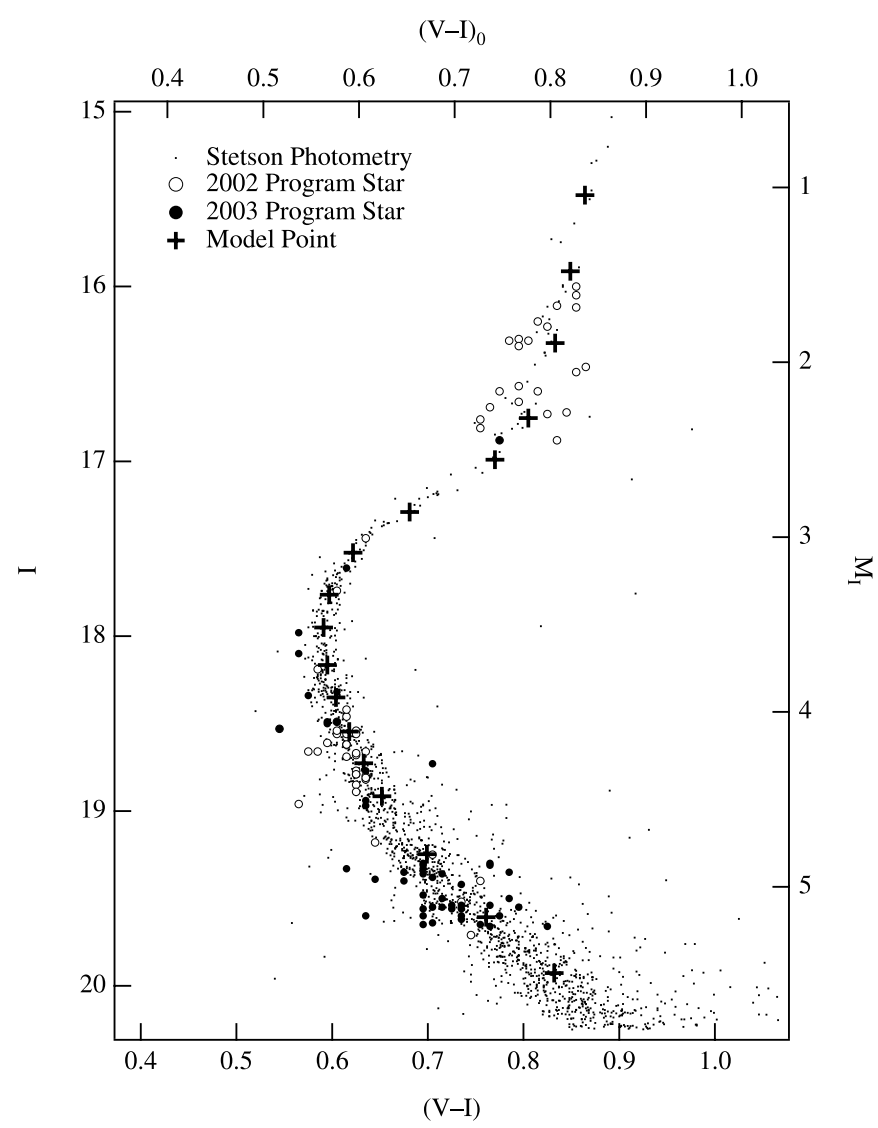

Fig. 1.-The $(I, V-I)$ color-magnitude diagram of M13 plotted using the database of P. B. S., with the locations of our program stars and those of BCS02 indicated. Also shown are the positions of the model points used in the present analysis.

and so was available for these observations. The high UV throughput of LRIS-B with this new, sensitive detector for the first time enabled us to reach the NH bands in the brighter of these stars with some precision. The locations of the faint program stars on the M13 color-magnitude diagram (CMD) are shown in Figure 1.

\subsection{The Bright Star Sample}

There are published surveys (Suntzeff 1981; Smith et al. $1996)$ in which $\mathrm{CH}$ indices have been used to determine $[\mathrm{C} / \mathrm{Fe}]$ values for large samples of the highest luminosity giants in M13. However, our Keck/LRIS sample of low-luminosity stars in M13 has no overlap with these earlier works. To ensure that the merger of our data for faint stars in M13 with these published data sets for $\mathrm{CH}$ band strengths in $\mathrm{M} 13$ giants is valid, we need to verify the consistency of the different measurements of the $\mathrm{CH}$ indices and resulting abundances. To demonstrate this, we obtained new blue spectra of a small sample of bright giants with published $\mathrm{CH}$ band strengths from earlier studies and remeasured their $\mathrm{CH}$ indices with the same procedures used for the lower luminosity M13 stars of our main sample (as described below). These spectra were taken in 2003 April and May at the Hale Telescope on Palomar Mountain during observing runs intended primarily for other projects. The blue channel of the Double Spectrograph (Oke \& Gunn 1982) was used with a 1200 line $\mathrm{mm}^{-1}$ grating and a Loral $512 \times 2788,15 \mu \mathrm{m}$ pixel CCD, giving $0.55 \AA \mathrm{pixel}^{-1}$ with a spectral resolution of $1.9 \AA$ for a $1^{\prime \prime}$ slit.

\section{ANALYSIS}

\subsection{The Faint Star Sample}

Our analysis essentially repeats that of BCS02 and is fully described in Briley \& Cohen (2001, hereafter BC01); the reader is referred to these works for details. To summarize, strengths of the $4350 \AA \mathrm{CH}(\mathrm{G})$ bands of our program stars were measured via the $I(\mathrm{CH})$ index of Cohen (1999a, 1999b) - an index that compares the flux removed by the $\mathrm{G}$ band with the adjacent continuum on both sides. The resulting indices, calculated using bandpasses corrected for the radial velocity of M13, are plotted for the sample of faint stars as a function of $I$ magnitude in Figure 2. The decrease in $\mathrm{CH}$ band strengths near $I \approx 18$ is due to the higher temperatures of the MSTO stars (as pointed out in $\mathrm{BC} 01$ ). However, among the fainter MS stars in the sample (near $I \approx 19.5$ ), the surface temperatures have dropped by roughly $300 \mathrm{~K}$ and again a large and significant scatter in $\mathrm{CH}$ band strengths is apparent. The $1 \sigma$ error bars plotted for the present sample have been determined entirely from Poisson statistics in the molecular band and continuum spectral windows.

In a similar manner, the strength of absorption by the $3350 \AA$ $\mathrm{NH}$ band was measured in spectra of the more luminous members of the Keck MS/MSTO sample using the doublesided logarithmic $s_{\mathrm{NH}}$ index as defined in Briley \& Smith (1993). The resulting indices (and $1 \sigma$ Poisson error bars) are also plotted in Figure 2. This marks the first time NH bands have been observed among such faint stars in a globular cluster. Spectra of two MSTO stars exhibiting differing NH band strengths, and two MS stars with differing $\mathrm{CH}$ band strengths, are shown in Figure 3.

In order to relate the observed indices to the underlying $[\mathrm{C} / \mathrm{Fe}]$, we employ a series of synthetic spectra based on MARCS (Gustafsson et al. 1975) model atmospheres. Our models are those used in $\mathrm{BC} 01$ and $\mathrm{BCS} 02$ and based on the $16 \mathrm{Gyr}[\mathrm{Fe} / \mathrm{H}]=-1.48$ O-enhanced isochrone grid of Bergbusch \& VandenBerg (2001). The locations of the model points on the M13 $(I, V-I)$ CMD are shown in Figure 1, assuming $(m-M)_{V}=14.43$ and a reddening of $E(B-V)=0.02$ as in $\mathrm{BC} 01$ and $\mathrm{BCS} 02$.

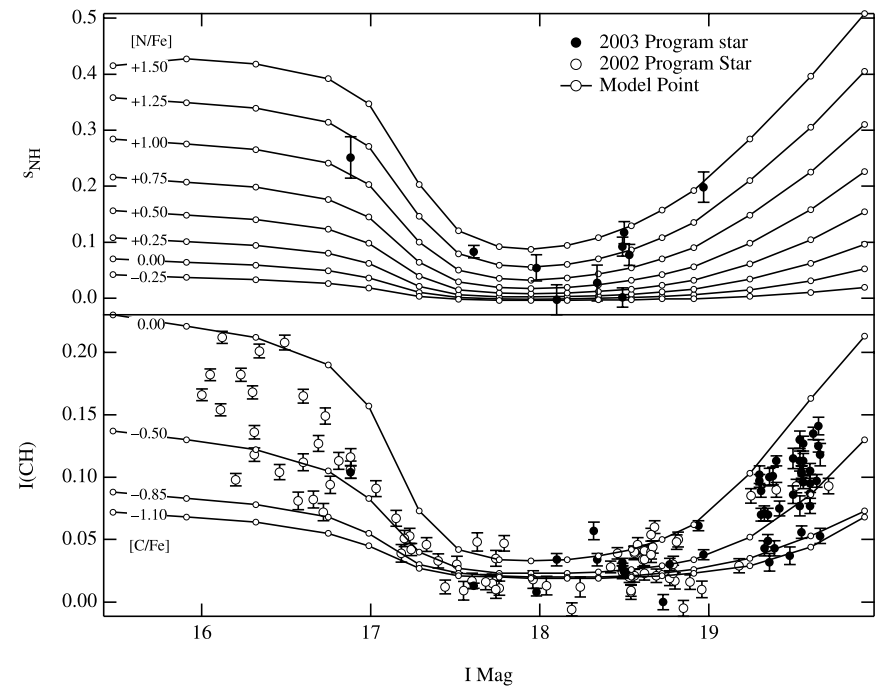

Fig. 2.-Bottom, G-band indices $[I(\mathrm{CH})]$ plotted as a function of $I$ for the program stars as well as those of BCS02; top, measured values of $s_{\mathrm{NH}}$. Error bars are $1 \sigma$ levels determined from Poisson statistics. Also plotted are model indices for a variety of $[\mathrm{C} / \mathrm{Fe}]$ and $[\mathrm{N} / \mathrm{Fe}]$ values as discussed in the text. 


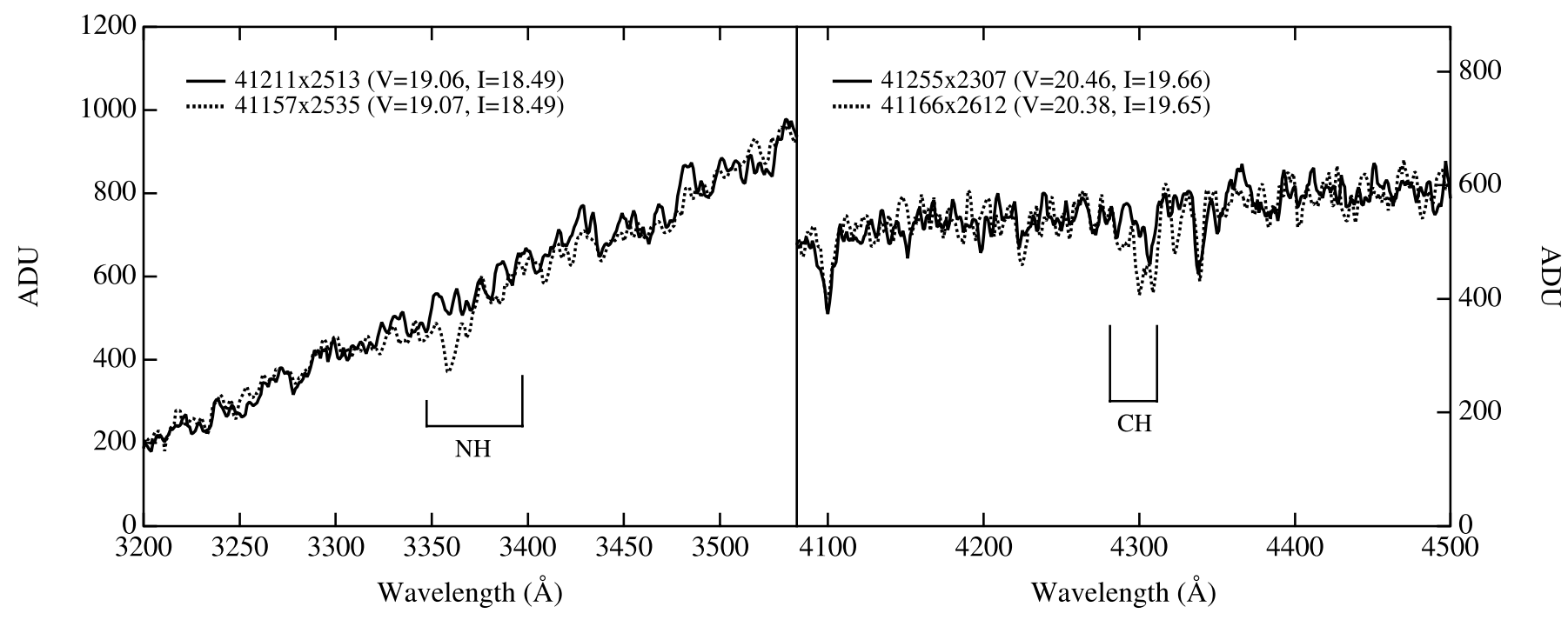

FIG. 3.- Sample spectra of the NH region of two similar M13 MSTO stars (left) and the G-band $(\mathrm{CH})$ region of two MS stars (right). In both, significant differences are apparent.

From each model and a given set of $\mathrm{C} / \mathrm{N} / \mathrm{O}$ abundances, synthetic spectra were computed using the SSG program (Bell, Paltoglou, \& Tripicco 1994 and references therein) and the line list of Tripicco \& Bell (1995) at a step size of $0.02 \AA$ (see BC01 for further details), assuming the average heavy-element compositions of Kraft et al. $(1993,1997)$. The result was then convolved with a Gaussian kernel to match the resolution of the observed spectra, and $I(\mathrm{CH})$ and $s_{\mathrm{NH}}$ indices were measured. The model indices for $I(\mathrm{CH})$ are illustrated in Figure 2 for four $\mathrm{C}$ abundances (as in $\mathrm{BCS} 02$ ): $[\mathrm{C} / \mathrm{Fe}]=-0.85$ and $[\mathrm{C} / \mathrm{Fe}]=-1.1$, which roughly match the observed compositions of M13's CN-weak and CN-strong bright giants, respectively (see Smith et al. 1996), and $[\mathrm{C} / \mathrm{Fe}]=0.0$ and $[\mathrm{C} / \mathrm{Fe}]=-0.5$. Also plotted in Figure 2 are $s_{\mathrm{NH}}$ indices for a variety of $[\mathrm{N} / \mathrm{Fe}]$ values. Note that among these relatively warm MS/MSTO stars, there is little sensitivity in the $\mathrm{CH}$ (NH) band strengths to changes in $\mathrm{N}, \mathrm{O}(\mathrm{C}, \mathrm{O})$ abundances (as opposed to the cool giants, where molecular equilibrium must be considered, particularly with regard to $\mathrm{O}$ ). As a check of this, Table 1 shows the sensitivity of $I(\mathrm{CH})$ and $s_{\mathrm{NH}}$ to such changes in a cool MS model $\left(T_{\text {eff }}=5601 \mathrm{~K}\right.$ and $\log g=4.66$, corresponding to an M13 MS star with $I=19.60$ ).

Following BCS02, we have applied the method of Briley et al. (1990) to convert the observed indices to corresponding $C$ and $\mathrm{N}$ abundances: the model isoabundance curves were interpolated to the $M_{I}$ of each program star, and the observed index was converted into the corresponding abundance based on the synthetic index at that $M_{I}$. Resulting $\mathrm{C}$ and $\mathrm{N}$ abundances are plotted in Figures 4 and 5. Note that the large error bars that accompany the stars of Figure 4 near $I=19$ and the stars of low

TABLE 1

Indices for Deviations from Assumed Composition for $T_{\text {eff }}=5601 \mathrm{~K}, \log g=4.66$ Model

\begin{tabular}{|c|c|c|c|c|}
\hline$[\mathrm{C} / \mathrm{Fe}]$ & {$[\mathrm{N} / \mathrm{Fe}]$} & {$[\mathrm{O} / \mathrm{Fe}]$} & $I(\mathrm{CH})$ & $s_{\mathrm{NH}}$ \\
\hline-0.50 & 0.0 & +0.40 & 0.075 & 0.044 \\
\hline-0.50 & +1.0 & +0.40 & 0.074 & 0.232 \\
\hline-0.50 & 0.0 & 0.00 & 0.079 & 0.048 \\
\hline-1.00 & 0.0 & +0.40 & 0.042 & 0.044 \\
\hline
\end{tabular}

$[\mathrm{C} / \mathrm{Fe}]$ (approximately -1 ) are due to the overall weakness of the $\mathrm{CH}$ bands - small errors in $I(\mathrm{CH})$ therefore result in large changes in $[\mathrm{C} / \mathrm{Fe}]$. Likewise, a similar situation exists among the MSTO stars with measured NH band strengths.

\subsection{The Bright Star Sample}

As with the faint stars, the $I(\mathrm{CH})$ index was measured from the spectra of the six bright M13 giants. For each star, this value was compared with synthetic indices generated from model atmospheres whose stellar parameters were taken from the high-resolution analyses of Kraft et al. $(1993,1997)$ and Pilachowski et al. (1996), including their heavy-element and $[\mathrm{O} / \mathrm{Fe}]$ abundances. Where available, $[\mathrm{N} / \mathrm{Fe}]$ values from Smith et al. (1996) were also used. For two stars (K188 and III-7), $\mathrm{N}$ abundances were not available from the literature, and a value of +1.0 was assumed. For star $\mathrm{III}-7$, an $[\mathrm{O} / \mathrm{Fe}]$ of 0.0 was used. The model parameters and the resulting $[\mathrm{C} / \mathrm{Fe}]$ abundance that matched the observed $I(\mathrm{CH})$ indices are listed

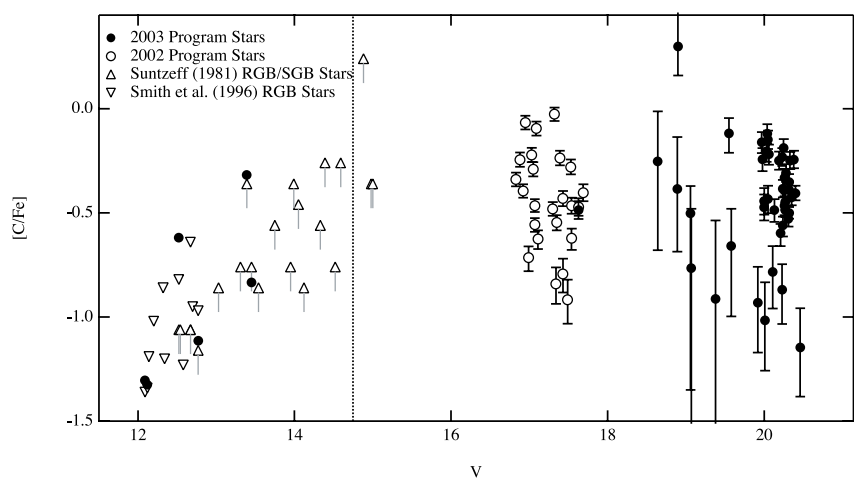

FIG. 4.-Derived $\mathrm{C}$ abundances for the present M13 MS and RGB stars, as well as the SGB stars of BCS02. Also plotted are the $[\mathrm{C} / \mathrm{Fe}]$ values from Smith et al. (1996) and Suntzeff (1981) (the latter having been shifted upward by 0.14 dex as discussed in the text; the size of this shift is indicated by the lines attached to the symbols). The dotted line indicates the location of the LF bump from Paltrinieri et al. (1998) - the point before which mixing is believed to be inhibited. There is a clear and significant scatter in $\mathrm{C}$ abundances among both the present MS sample and the SGB stars of BCS02. [C/Fe] appears to decrease with $V$ among the most luminous giants as would be expected from mixing, but the onset is uncertain. 


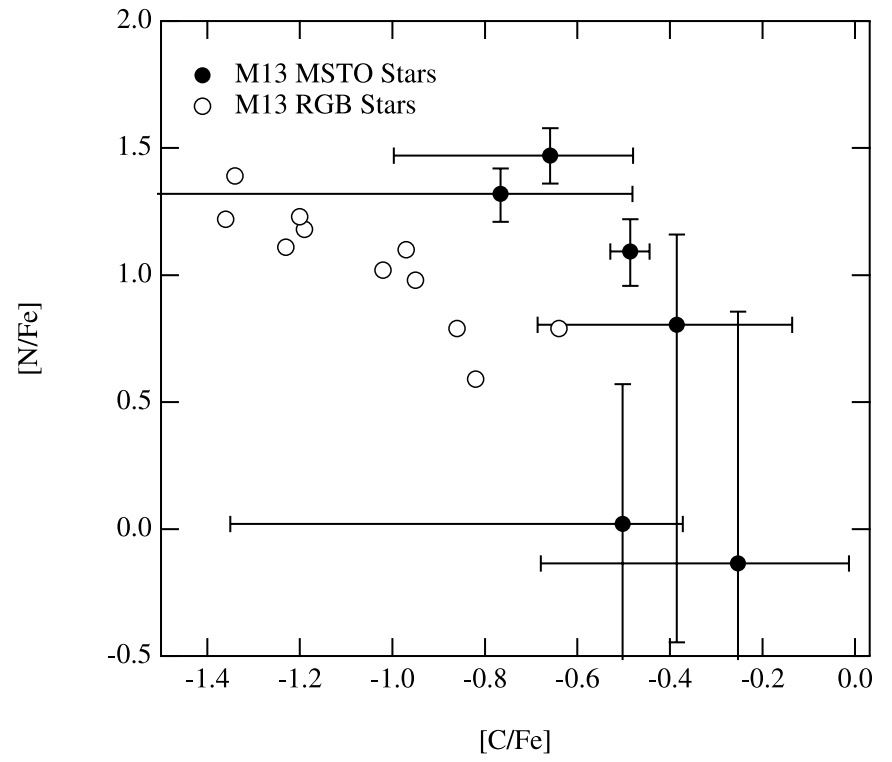

Fig. 5.-Values of [N/Fe] plotted vs. [C/Fe] for the M13 MSTO stars where (despite the large error bars) an anticorrelation is suggested. Also shown are the abundances from luminous giants from Smith et al. (1996), which, as expected from Fig. 4, appear more deficient in $[\mathrm{C} / \mathrm{Fe}]$. That the presumably mixed RGB stars do not show greater $\mathrm{N}$ abundances than their MSTO counterparts appears to be the result of the large initial $\mathrm{N}$ abundances, as discussed in the text.

in Table 2 along with the $\mathrm{C}$ abundances from Smith et al. (1996) and Suntzeff (1981).

For the four bright giants in common with Smith et al. (1996), we find an average offset of $0.03( \pm 0.14)$ dex in $[\mathrm{C} / \mathrm{Fe}]$ (present minus Smith). We therefore consider our $\mathrm{C}$ abundances to be essentially on the same scale, as might be expected considering the similar analysis tools used. The difference between our results and those of Suntzeff (1981) are somewhat larger: $0.25( \pm 0.23)$. However, almost half of this offset is driven by the result for II-76. Excluding this star reduces the average difference to $0.14( \pm 0.07)$. Note that II-76 has both a high $[\mathrm{O} / \mathrm{Fe}]$ and a lower $[\mathrm{N} / \mathrm{Fe}]$ abundance, as might be expected from a star with a lesser amount of $\mathrm{CN}(\mathrm{O})$ cycle material in its atmosphere (it also has the second lowest $\mathrm{Na}$ abundance of the large sample of Pilachowski et al. 1996). The source of this discrepancy is likely the cooler model used for II- 76 by Suntzeff ( $T_{\text {eff }}=4220 \mathrm{~K}$, vs. the $4350 \mathrm{~K}$ used here), as well as the lower $\mathrm{O}$ abundance $([\mathrm{O} / \mathrm{Fe}]=0.0)$ and higher microturbulent velocity $\left(2.5 \mathrm{~km} \mathrm{~s}^{-1}\right)$. Repeating our analysis with the values used by Suntzeff reduces our resulting $[\mathrm{C} / \mathrm{Fe}]$ by 0.32 dex to -0.96 . The luminous stars of Suntzeff plotted in Figure 4 have therefore been shifted by 0.14 in $[\mathrm{C} / \mathrm{Fe}]$ to place them on our abundance scale.

Given the use of the same modeling codes, line lists, and $\mathrm{CH}$ indices throughout our analysis, we presume the resulting $\mathrm{C}$ abundances from both the faint and bright star samples, as well as those of Smith et al. (1996) and Suntzeff (1981; with the appropriate shift), to be on the same abundance scale. Any systematic differences due to different telescope-spectrograph systems will be minimized by the use of the $I(\mathrm{CH})$ index, which uses continuum bands both blueward and redward of the $\mathrm{CH}$ feature to remove slope differences due to variations in instrumental response.

\section{RESULTS}

There are several points to be made about the present results, which are given in tabular form in Tables 3 and 4. First, as can be seen in Figure 2, significant differences in $[\mathrm{C} / \mathrm{Fe}]$ exist among stars at least 1.5 mag fainter than the MSTO in M13. This corresponds to a mass of approximately $0.66 M_{\odot}$ using the isochrone of Figure 1. Among these old MS stars, $\mathrm{CN}(\mathrm{O})$-cycle reactions are entirely confined to the central core (see, e.g., Figs. 4 and 5 of Richard et al. 2002), and as has been pointed out by numerous investigators, MS stars such as these are not thought to possess a mechanism that connects their surface with regions of energy generation (namely, the core). Indeed, should such mixing take place, the subsequent paths of the stars in the CMD would be radically altered by the infusion of fresh $\mathrm{H}$ into the core (see, e.g., VandenBerg \& Smith 1988). One must conclude the source of the observed differences in $[\mathrm{C} / \mathrm{Fe}]$ is likely not the stars themselves. Moreover, the values of $[\mathrm{C} / \mathrm{Fe}]$ among the MS stars are consistent with those found by BCS02 among the M13 SGB stars (see Fig. 4) and imply that little change in composition has occurred from the MS to at least the base of the SGB.

Figure 4 also includes the $[\mathrm{C} / \mathrm{Fe}]$ values of Smith et al. (1996) and Suntzeff (1981; shifted upward by 0.14 dex). As discussed in $\mathrm{BCS} 02$, there appears to be a marked decline in $[\mathrm{C} / \mathrm{Fe}]$ toward higher luminosities among the M13 giants. Clearly an evolutionary change such as this can be best interpreted as the result of a mixing process bringing up C-depleted material from a region in which at least $\mathrm{CN}$-cycle reactions are operating (see BCS02 for a more detailed discussion). Also shown in Figure 4 is the location of the LF bump in M13 (from Paltrinieri et al. 1998) - the event that marks the destruction of the molecular-weight gradient thought to inhibit deep mixing. Unfortunately, the luminosity at which the onset of C depletion begins is uncertain because of the gap in the available data (in the range $15<V<17$ ). However, since an extrapolation of the trend in giant branch $[\mathrm{C} / \mathrm{Fe}]$ abundance faintward

TABLE 2

Indices, Model Atmosphere Parameters, and Resulting [C/Fe] Abundances for M13 Bright Giants

\begin{tabular}{|c|c|c|c|c|c|c|c|c|c|c|}
\hline \multirow[b]{2}{*}{ STAR } & \multirow[b]{2}{*}{$I(\mathrm{CH})$} & \multirow{2}{*}{$\begin{array}{l}T_{\text {eff }} \\
(\mathrm{K})\end{array}$} & \multirow[b]{2}{*}{$\log g$} & \multirow{2}{*}{$\begin{array}{c}v_{t} \\
\left(\mathrm{~km} \mathrm{~s}^{-1}\right)\end{array}$} & \multirow{2}{*}{$\begin{array}{c}V \\
(\mathrm{mag})\end{array}$} & \multirow[b]{2}{*}[\mathrm{O}/\mathrm{Fe}]{} & \multirow[b]{2}{*}[\mathrm{N}/\mathrm{Fe}]{} & \multicolumn{3}{|c|}{$[\mathrm{C} / \mathrm{Fe}]$} \\
\hline & & & & & & & & Present & Smith et al. 1996 & Suntzeff 1981 \\
\hline 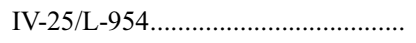 & 0.166 & 4000 & 0.15 & 2.25 & 12.09 & -0.90 & +1.22 & -1.31 & -1.36 & $\ldots$ \\
\hline II-67/L-70 ......................................... & 0.165 & 3950 & 0.20 & 2.10 & 12.12 & -0.79 & +1.33 & -1.32 & -1.34 & $\ldots$ \\
\hline 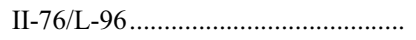 & 0.200 & 4350 & 1.15 & 1.85 & 12.52 & +0.46 & +0.59 & -0.62 & -0.82 & -1.2 \\
\hline 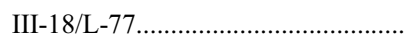 & 0.156 & 4350 & 1.15 & 1.85 & 12.77 & -0.18 & +1.10 & -1.11 & -0.97 & -1.3 \\
\hline 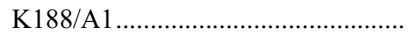 & 0.238 & 4550 & 1.50 & 1.80 & 13.39 & +0.45 & +1.00 & -0.32 & $\ldots$ & -0.5 \\
\hline 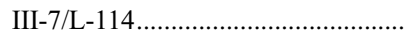 & 0.173 & 4600 & 1.65 & 2.00 & 13.45 & 0.00 & +1.00 & -0.83 & $\ldots$ & -0.9 \\
\hline
\end{tabular}


TABLE 3

Current Program Stars: Photometry, Indices, and Abundances

\begin{tabular}{|c|c|c|c|c|c|c|c|}
\hline Star & $V$ & $I$ & $V-I$ & $I(\mathrm{CH})$ & $s_{\mathrm{NH}}$ & {$[\mathrm{C} / \mathrm{Fe}]$} & {$[\mathrm{N} / \mathrm{Fe}]$} \\
\hline 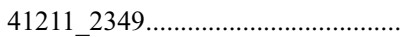 & 17.30 & 17.10 & 0.20 & -0.010 & -0.024 & $\ldots$ & $\ldots$ \\
\hline 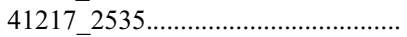 & 17.63 & 16.88 & 0.75 & 0.104 & 0.228 & -0.49 & +1.09 \\
\hline 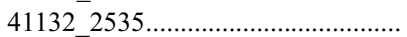 & 18.20 & 17.61 & 0.59 & 0.013 & 0.060 & $\ldots$ & +1.37 \\
\hline 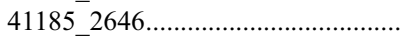 & 18.52 & 17.98 & 0.54 & 0.008 & 0.031 & $\ldots$ & +1.24 \\
\hline 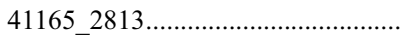 & 18.64 & 18.10 & 0.54 & 0.034 & -0.026 & -0.25 & -0.14 \\
\hline
\end{tabular}

Noте.-Table 3 is presented in its entirety in the electronic edition of the Astronomical Journal. A portion is shown here for guidance regarding its form and content.

intersects the magnitude of the LF bump at the average abundance of the fainter stars, it is reasonable to infer that the abundance decline begins near that event; neither a significant decrease nor a significant increase in $\mathrm{C}$ abundance with a subsequent recovery to the original value hidden within the gap in our data is reasonably to be expected.

The $[\mathrm{N} / \mathrm{Fe}]$ values determined from the $\mathrm{NH}$ band strengths of the MSTO stars are plotted in Figure 5. Although the error bars are admittedly larger than one would like, owing to the weaknesses of the $\mathrm{CH}$ and $\mathrm{NH}$ bands among the warmer MSTO stars, a general anticorrelation between $[\mathrm{C} / \mathrm{Fe}]$ and $[\mathrm{N} / \mathrm{Fe}]$ is suggested. Note that these abundances do not suffer from the inherent tendency toward C-N anticorrelations of analyses based on $\mathrm{CH}$ and $\mathrm{CN}$ band strengths. Of course an overall C-N anticorrelation is known to be present among the evolved M13 stars, and the values for the bright RGB stars of Smith et al. (1996) are also shown in Figure 5. Immediately apparent is the shift of the RGB stars toward lower $[\mathrm{C} / \mathrm{Fe}]$, as is expected from Figure 4. If C-poor/N-rich material is indeed being circulated into the stellar envelopes during RGB ascent, the lack of nearsolar [N/Fe] RGB stars is also explained (although the error bars on the two lower $[\mathrm{N} / \mathrm{Fe}]$ MSTO stars severely limit the weight that can be placed on this statement). That higher $\mathrm{N}$ abundances do not appear to be found among the RGB stars under these circumstances is perhaps not a surprise if these stars are already leaving the MSTO with large $[\mathrm{N} / \mathrm{Fe}]$ overabundances: an M13 MSTO star with $[\mathrm{C} / \mathrm{Fe}]=-0.4$ and $[\mathrm{N} / \mathrm{Fe}]=1.0$ that undergoes a mixing episode reducing $[\mathrm{C} / \mathrm{Fe}]$ to -1.2 will experience a rise in $[\mathrm{N} / \mathrm{Fe}]$ of only $0.05 \mathrm{dex}$-in essence, the $\mathrm{N}$ abundances are already so large that the addition of freshly minted $\mathrm{N}$ via the $\mathrm{CN}$ cycle results in only a small fractional change in $[\mathrm{N} / \mathrm{Fe}]$. Thus, while the error bars in Figure 5 are large, we can at least claim that it is not inconsistent with the assertion that we are seeing substantial star-to-star variations in $\mathrm{C}$ (and $\mathrm{N}$ ) set early in the cluster history, which are further being modified by mixing during RGB ascent. The possibility of also mixing $\mathrm{ON}$-cycle material to the surface is more difficult to assess because of the large $\mathrm{N}$ variations among the MSTO stars. In the example above, an additional reduction in $[\mathrm{O} / \mathrm{Fe}]$ from +0.45 to -0.35 would increase $[\mathrm{N} / \mathrm{Fe}$ ] by $0.46 \mathrm{dex}$ - and among the bright giants, even larger $\mathrm{O}$ depletions (as much as $[\mathrm{O} / \mathrm{Fe}]=-0.7$ to -0.8$)$ have been noted. Starting with an even larger $\mathrm{N}$ overabundance of +1.4 reduces the change in [N/Fe] to +0.25 . However, at least from the small sample of Figure 5, it appears that none of the bright RGB stars possess larger $\mathrm{N}$ abundances than their MSTO counterparts, which in turn suggests the envelopes of at least the initially N-rich stars may not be cycled through a region of ON-cycle reactions while on the RGB. Clearly, knowledge of the $\mathrm{O}$ abundances of the M13 MSTO stars would help settle this question.

A similar result was noted in the more metal-poor clusters M92 and M15 by Carbon et al. (1982) and Trefzger et al. (1983), respectively - that substantial N overabundances are present from the SGB to the AGB that are not necessarily correlated with $\mathrm{C}$ abundances. Indeed, an analogous situation can been seen in the present results and those of Cohen et al. (2002) for M5 (see Figs. 5 and 6): the "higher" [C/Fe] MSTO stars (at approximately -0.4 ) span almost a dex in $[\mathrm{N} / \mathrm{Fe}]$. It is clear that if we are to ascribe the same mechanism to the origin of the SGB/MSTO inhomogeneities in these clusters, it must be operating at the MSTO or earlier.

\section{DISCUSSION}

That significant and correlated star-to-star differences in $\mathrm{C}$ and $\mathrm{N}$, as well as $\mathrm{O}, \mathrm{Na}, \mathrm{Al}$, and $\mathrm{Mg}$, have been found among the SGB, MSTO, and MS stars of several clusters (see references above) implies the operation of some process external to the present stars, presumably having taken place early in the cluster history. The discussion by Cannon et al. (1998) includes a comprehensive look at various possibilities. It is worthwhile, however, to revisit a few of the more critical constraints on any theory of the origin of the abundance variations.

TABLE 4

M5 Subgiants from BCS02: Program Stars, Photometry, Indices, and Abundances

\begin{tabular}{|c|c|c|c|c|c|}
\hline Star & V & $I$ & $V-I$ & $I(\mathrm{CH})$ & {$[\mathrm{C} / \mathrm{Fe}]$} \\
\hline 41230_2604...... & 16.83 & 16.00 & 0.83 & 0.166 & -0.34 \\
\hline 41244_2423...................................... & 16.88 & 16.05 & 0.83 & 0.182 & -0.25 \\
\hline 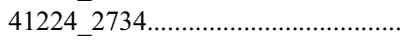 & 16.92 & 16.11 & 0.81 & 0.154 & -0.40 \\
\hline 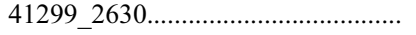 & 16.95 & 16.12 & 0.83 & 0.212 & -0.07 \\
\hline 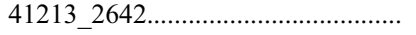 & 16.99 & 16.20 & 0.79 & 0.098 & -0.72 \\
\hline
\end{tabular}

Note.-Table 4 is presented in its entirety in the electronic edition of the Astronomical Journal. A portion is shown here for guidance regarding its form and content.

${ }^{a}$ Values of $[\mathrm{C} / \mathrm{Fe}]$ were not determined for stars near the MSTO, because of the weakness of the $\mathrm{CH}$ bands. 


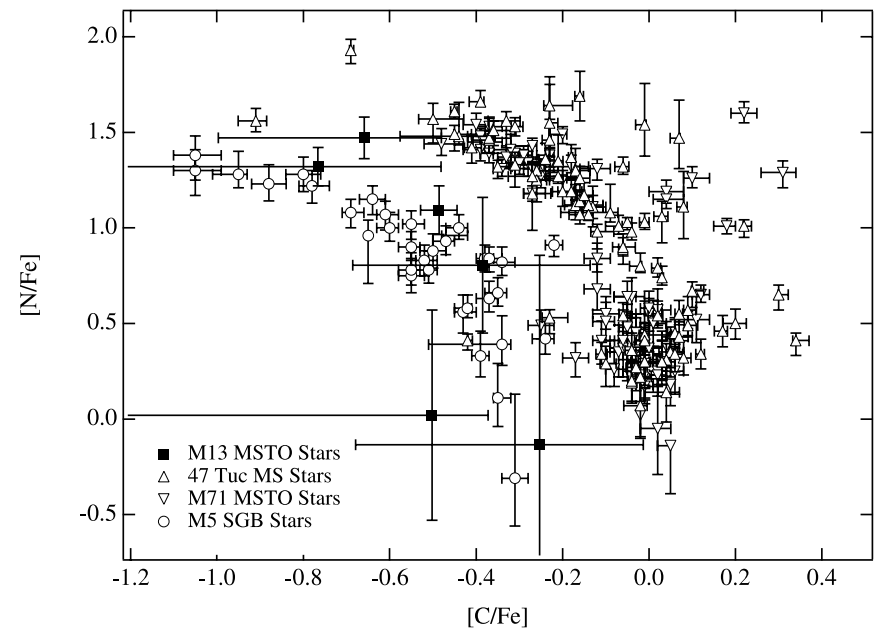

FIG. 6. - The $[\mathrm{N} / \mathrm{Fe}]$ vs. [C/Fe] values plotted for MS, MSTO, and SGB stars in four different clusters. The present MSTO abundances appear consistent with the SGB stars of M5 (Cohen et al. 2002), a cluster of roughly similar metallicity $([\mathrm{Fe} / \mathrm{H}]=-1.26$ vs. -1.51$)$, as opposed to those of the higher metallicity 47 Tuc and M71 stars $([\mathrm{Fe} / \mathrm{H}]=-0.7)$.

First, whatever mechanism has altered the light-element compositions of the cluster stars has left the heavy elements essentially untouched, at least to the limits of our ability to determine them - the analysis of M5 by Ramírez \& Cohen (2003) is an excellent example. This alone would seem to exclude the possibility of the light-element variations arising from the merger of two distinct protocluster clouds (as has been pointed out by numerous authors).

Second, these abundance variations appear to be almost ubiquitous among the population of Galactic globular clusters (GCs). To highlight this, we have plotted in Figure 6 the $[\mathrm{C} / \mathrm{Fe}]$ and $[\mathrm{N} / \mathrm{Fe}]$ values for the present sample of M13 MSTO stars, the 47 Tuc MS stars of Briley et al. (2004), the M5 SGB stars of Cohen et al. (2002), and the MSTO stars of M71 from $\mathrm{BC} 01$. Note that $\mathrm{BC} 01$ did not directly extract $\mathrm{C}$ and $\mathrm{N}$ abundances from their observed indices-we have converted them here following the procedure outlined in Cohen et al. (2002) and using the indices and models presented in BC01; the values are given in Table 5 .

Third, the elements that are observed to vary are associated with proton-capture nucleosynthesis under conditions of $\mathrm{CN}$ and ON cycling. The source or site must process these CNOgroup elements and return this material to the cluster to be incorporated into the present population of low-mass stars either before, during, or after their formation.

A popular model that fits these constraints is the incorporation of ejecta from intermediate-mass $\left(3-6 M_{\odot}\right)$ AGB stars undergoing hot bottom burning and third dredge-up (see
Ventura et al. 2001), although difficulties, such as the establishment of an $\mathrm{O}-\mathrm{Na}$ anticorrelation, remain (see, e.g., Denissenkov \& Herwig 2003). However, as is discussed in Briley, Smith, \& Claver (2001) and BCS02, the quantities of material required to produce the observed star-to-star differences among the low-luminosity stars (most notably extreme $\mathrm{C}$ depletions), which are clearly not diluted as the convective envelopes deepen during RGB ascent, rule out any sort of simple accretion model. Indeed, for the present M13 stars, roughly $70 \%$ of a C-poor MS star's total mass must be captured ejecta if the accreted matter is completely free of $\mathrm{C}$ (see BCS02). It is of course not clear how such an enormous amount of material can be returned to the cluster without appealing to a shallow initial mass function (see Briley et al. 2001), or how the present stars can sweep up the necessary mass of ejecta (although a novel look at accretion by Thoul et al. 2002 suggests significant quantities of AGB ejecta could be captured by stars in clusters with high central concentrations, it should be noted that M13 is definitely not a cluster with a high central concentration). We note that in Figure 6 the depletions in $\mathrm{C}$ do appear smaller in the more metal-rich clusters M71 and 47 Tuc, in accord with the prediction of the AGB ejecta models of Ventura et al. (2001). Yet at the same time, if one presumes the highest [C/Fe] SGB/MSTO stars in M13 and M5 to represent the original (accretion-free) C abundance of the cluster stars, they are still some 0.4 dex more C-poor than their 47 Tuc/M71 counterparts, implying either truly primordial (i.e., preaccretion) differences in at least $\mathrm{C}$ or that nearly all of the present stars in M13 and M5 have undergone at least some accretion of C-poor material. However, the spread in $[\mathrm{N} / \mathrm{Fe}]$ is essentially identical among all four clusters. Clearly, knowledge of the patterns of $[\mathrm{O} / \mathrm{Fe}]$ and $[\mathrm{Na} / \mathrm{Fe}]$ among the present stars would help constrain the AGB ejecta theories.

An interesting counterpoint to this model is the scenario suggested by Carbon et al. (1982) and Trefzger et al. (1983) to explain similar results among M92 and M15 SGB stars- that the stars of these clusters were inhomogeneously "polluted" by an injection of raw $\mathrm{C}$ from intermediate-mass AGB stars that is subsequently converted into $\mathrm{N}$ in the present stars before SGB evolution, thereby explaining both the $\mathrm{C}$ deficiencies and large $\mathrm{N}$ enhancements, as well as star-to-star differences in $\mathrm{C}+\mathrm{N}$. This has the additional advantage of requiring considerably more modest composition modifications (a factor of 4 or so in C from star to star), which in turn lowers the mass of captured ejecta required. However, to explain the large $\mathrm{C}$ depletions already in place by the MSTO, significant processing of the envelope through a region of $\mathrm{CN}$ cycling must have taken place while the stars occupied the MS. One then returns to the difficulty of mixing in such stars discussed above.

TABLE 5

M71 Subgiants from BC01: Program Stars, Photometry, Indices, and Abundances

\begin{tabular}{|c|c|c|c|c|c|c|}
\hline Star & $R$ & $B-R$ & $I(\mathrm{CH})$ & $S(3839)$ & {$[\mathrm{C} / \mathrm{Fe}]$} & {$[\mathrm{N} / \mathrm{Fe}]$} \\
\hline 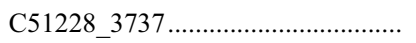 & 17.00 & 1.38 & 0.138 & 0.131 & -0.17 & +0.32 \\
\hline 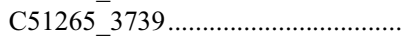 & 17.01 & 1.31 & 0.122 & 0.141 & -0.25 & +0.49 \\
\hline 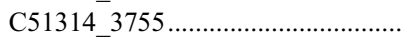 & 17.01 & 1.34 & 0.096 & 0.340 & -0.39 & +1.41 \\
\hline 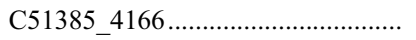 & 17.01 & 1.40 & 0.092 & 0.378 & -0.40 & +1.54 \\
\hline 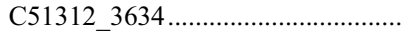 & 17.03 & 1.35 & 0.156 & 0.388 & +0.04 & +1.19 \\
\hline
\end{tabular}

Note.-Table 5 is presented in its entirety in the electronic edition of the Astronomical Journal. A portion is shown here for guidance regarding its form and content. 
TABLE 6

Precision Radial Velocities for Three M5 Stars

\begin{tabular}{|c|c|c|c|}
\hline Star & $\begin{array}{c}V \\
(\mathrm{mag})\end{array}$ & $\begin{array}{c}\text { R.V. } \\
\left(\mathrm{km} \mathrm{s}^{-1}\right)\end{array}$ & Comment \\
\hline C182250537 … & 17.07 & +58.2 & Anomalous star in Cohen et al. 2002 \\
\hline 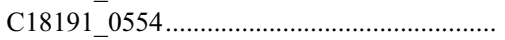 & 17.12 & +63.1 & In LRIS sample, but not anomalous \\
\hline 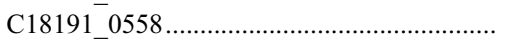 & 17.57 & +58.5 & Anomalous in Cohen et al. 2002 as C18211_0559 \\
\hline
\end{tabular}

Another site of the proton-capture reactions has recently been suggested by Li \& Burstein (2003), who note that the high-mass $\left(250-300 M_{\odot}\right)$, zero-metallicity models of Fryer, Woosley, \& Heger (2001) tend to mix He and He burning products into their H-burning shells during the later stages of He burning. This fresh $\mathrm{C}, \mathrm{N}$, and $\mathrm{O}$ is partially processed into $\mathrm{N}$, while at the same time the stars expand into red supergiants. If mass loss also occurs at this point, the cluster could be seeded with freshly produced $\mathrm{C} / \mathrm{O}$-poor, $\mathrm{N}$-rich material. Such a scenario is presented within the context of the cluster formation history of Cayrel (1986) - that the GCs formed from primordial material (zero metal) that was subsequently enriched by the supernovae of massive stars before low-mass stars could form. However, the problem remains that the production or seeding and mixing of the heavy elements must be decoupled from that of the light elements in order to explain the remarkable homogeneity of $\mathrm{Fe}, \mathrm{Ti}, \mathrm{Ca}$, etc., within the GCs. In the context of GC formation in a well-mixed supershell (see, e.g., Brown, Burkert, \& Truran 1991), this is difficult to explain if the CNO-modified material is ejected prior to the driving supernovae and subsequent supershell expansion/mixing.

The entire Keck/LRIS user community owes a huge debt to Jerry Nelson, Gerry Smith, Bev Oke, and many other people who have worked to make the Keck Telescope and LRIS a reality. We are grateful to the W. M. Keck Foundation, and particularly its late president, Howard Keck, for the vision to fund the construction of the W. M. Keck Observatory. We also wish to express our thanks to Roger Bell, whose SSG code was instrumental in this project, and the anonymous referee for suggestions. Partial support was provided by the National Science Foundation under grant AST 00-98489 to M. M. B. and grant AST 02-05951 to J. G. C. and by the F. John Barlow Professorship and University of Wisconsin Oshkosh Faculty Development Program (M. M. B.).

\section{APPENDIX}

\section{UPDATE ON ANOMALOUS STARS PREVIOUSLY OBSERVED IN M5}

In Cohen et al. (2002), we studied the $\mathrm{CH}$ bands in a large sample of stars in M5. Even taking into account the substantial star-to-star variation seen among the $\mathrm{CH}$ band strengths of the stars in our sample, we denoted six of these stars as anomalous. Since that time, we have checked the data for these stars yet again. We have found that two of the six stars were misidentified. C18206_0533, with $V=18.42$, is actually C18188_0733, with $(B, V, I)=(17.71,17.03,16.17)$. With this correction, as compared wish the bulk of our M5 sample (see Figs. 8 and 9 of Cohen et al. 2002) the star has normal $\mathrm{CH}$ for its $T_{\text {eff }}$, although its uvCN is still anomalously strong, but not as much as previously. Also, star C18211_0559 $(V=18.06)$ is actually C18191_0559, with $(B, V, I)=(\overline{18.27}, 17.57,16.74)$. Its $\mathrm{CN}$ is now reasonable for its corrected $V$ magnitude, but its $\mathrm{CH}$ index is still unexpectedly strong.

In our earlier paper, we presented low-accuracy radial velocities from the LRIS spectra at $\mathrm{H} \alpha$, which suggested that four of the six stars classified as anomalous are radial velocity members of M5. Such data were not available for one star, while the radial velocity of C18211_0559 (now identified as C18191_0559) was $25 \mathrm{~km} \mathrm{~s}^{-1}$ higher than that of the cluster.

To verify the membership of C18191_0559 in M5, we obtained low signal-to-noise ratio spectra with HIRES (Vogt et al. 1994) for it and for a second star from the LRIS sample. A single $1200 \mathrm{~s}$ exposure for each was made on 2002 May 1, a night with considerable clouds. The HIRES slit for one of these two also included a second M5 star. The heliocentric radial velocities for these three stars derived from the $\mathrm{Na} D$ lines are presented in Table 6 . The radial velocity for M5 found by Ramírez \& Cohen (2002) from an extensive highdispersion analysis of stars over a wide range in luminosity is $+55.0 \mathrm{~km} \mathrm{~s}^{-1}$, so we conclude that all three of these stars are members.
Bell, R. A., Paltoglou, G., \& Tripicco, M. J. 1994, MNRAS, 268, 771

Bergbusch, P. A., \& VandenBerg, D. A. 2001, ApJ, 556, 322

Briley, M. M., Bell, R. A., Hoban, S., \& Dickens, R. J. 1990, ApJ, 359, 307

Briley, M. M., \& Cohen, J. G. 2001, AJ, 122, 242 (BC01)

Briley, M. M., Cohen, J. G., \& Stetson, P. B. 2002, ApJ, 579, L17 (BCS02)

Briley, M. M., Harbeck, D., Smith, G. H., \& Grebel, E. K. 2004, AJ, 127,1588

Briley, M. M., \& Smith, G. H. 1993, PASP, 105, 1260

Briley, M. M., Smith, G. H., \& Claver, C. F. 2001, AJ, 122, 2561

Briley, M. M., Smith, V. V., Suntzeff, N. B., Lambert, D. L., Bell, R. A., \& Hesser, J. E. 1996, Nature, 383, 604

Brown, J. H., Burkert, A., \& Truran, J. W. 1991, ApJ, 376, 115

Cannon, R. D., Croke, B. F. W., Bell, R. A., Hesser, J. E., \& Stathakis, R. A. 1998, MNRAS, 298, 601

Carbon, D. F., Langer, G. E., Butler, D., Kraft, R. P., Suntzeff, N. B., Kemper, E., Trefzger, C. F., \& Romanishin, W. 1982, ApJS, 49, 207

\section{REFERENCES}

Cavallo, R. M., Sweigart, A. V., \& Bell, R. A. 1996, ApJ, 464, L79

Cayrel, R. 1986, A\&A, 168, 81

Charbonnel, C., Brown, J. A., \& Wallerstein, G. 1998, A\&A, 332, 204

Cohen, J. G. 1999a, AJ, 117, 2428 1999b, AJ, 117, 2434

Cohen, J. G., Briley, M. M., \& Stetson, P. B. 2002, AJ, 123, 2525

Da Costa, G. S. 1998, in IAU Symp. 189, Fundamental Stellar Properties, ed. T. R. Bedding, A. J. Booth, \& J. Davis (Dordrecht: Kluwer), 193

Denissenkov, P. A., \& Denissenkova, S. N. 1990, AZh Pisma, 16, 642 (English transl. Soviet Astron. Lett, 16, 275)

Denissenkov, P. A., \& Herwig, F. 2003, ApJ, 590, L99

Denissenkov, P. A., \& VandenBerg, D. A. 2003, ApJ, 593, 509

Fryer, C. L., Woosley, S. E., \& Heger, A. 2001, ApJ, 550, 372

Gratton, R. G., et al. 2001, A\&A, 369, 87

Grundahl, F., Briley, M., Nissen, P. E., \& Feltzing, S. 2002, A\&A, 385, L14

Gustafsson, B., Bell, R. A., Eriksson, K., \& Nordlund, Å. 1975, A\&A, 42, 407 
Harbeck, D., Smith, G. H., \& Grebel, E. K. 2003, AJ, 125, 197

Hesser, J. E. 1978, ApJ, 223, L117

Kraft, R. P. 1994, PASP, 106, 553

Kraft, R. P., Sneden, C., Langer, G. E., \& Shetrone, M. D. 1993, AJ, 106, 1490

Kraft, R. P., Sneden, C., Smith, G. H., Shetrone, M. D., \& Fulbright, J. 1998, AJ, 115,1500

Kraft, R. P., Sneden, C., Smith, G. H., Shetrone, M. D., Langer, G. E., \& Pilachowski, C. A. 1997, AJ, 113, 279

Langer, G. E., \& Hoffman, R. D. 1995, PASP, 107, 1177

Li, Y., \& Burstein, D. 2003, ApJ, 598, L103

McCarthy, J. K., et al. 1998, Proc. SPIE, 3355, 81

Oke, J. B., et al. 1995, PASP, 107, 375

Oke, J. B., \& Gunn, J. E. 1982, PASP, 94, 586

Paltrinieri, B., Ferraro, F. R., Fusi Pecci, F., \& Carretta, E. 1998, MNRAS, 293, 434

Pilachowski, C. A., Sneden, C., Kraft, R. P., \& Langer, G. E. 1996, AJ, 112,545

Ramírez, S. V., \& Cohen, J. G. 2002, AJ, 123, 3277 2003, AJ, 125, 224

Richard, O., Michaud, G., Richer, J., Turcotte, S., Turck-Chièze, S., \& VandenBerg, D. A. 2002, ApJ, 568, 979
Shetrone, M. D. 2003, ApJ, 585, L45

Shortridge, K. 1988, Figaro: A General Data Reduction System (ver. 2.4; Epping: Anglo-Australian Obs.)

Smith, G. H. 1987, PASP, 99, 67

Smith, G. H., Shetrone, M. D., Bell, R. A., Churchill, C. W., \& Briley, M. M. 1996, AJ, 112, 1511

Stetson, P. B. 2000, PASP, 112, 925

Stetson, P. B., Hesser, J. E., \& Smecker-Hane, T. A. 1998, PASP, 110, 533

Suntzeff, N. B. 1981, ApJS, 47, 1

Suntzeff, N. B., \& Smith, V. V. 1991, ApJ, 381, 160

Sweigart, A. V., \& Mengel, J. G. 1979, ApJ, 229, 624

Thoul, A., Jorissen, A., Goriely, S., Jehin, E., Magain, P., Noels, A., \& Parmentier, G. 2002, A\&A, 383, 491

Trefzger, C. F., Carbon, D. F., Langer, G. E., Suntzeff, N. B., \& Kraft, R. P. 1983, ApJ, 266, 144

Tripicco, M. J., \& Bell, R. A. 1995, AJ, 110, 3035

VandenBerg, D. A., \& Smith, G. H. 1988, PASP, 100, 314

Ventura, P., D'Antona, F., Mazzitelli, I., \& Gratton, R. 2001, ApJ, 550, L65

Vogt, S. S., et al. 1994, Proc. SPIE, 2198, 362 